\title{
Haftung des Krankenhausträgers für Fehler eines Mitarbeiters bei Errichtung eines Nottestaments
}

In seinem Urteil vom 13.04.2010 bejaht das OLG Hamm (A2.: I-21 U 94/09) die Schadensersatzpflicht eines Krankenhauses gegenüber der Lebensgefährtin eines Patienten, die aufgrund eines von einem Krankenhausmitarbeiter aufgesetzten, unwirksamen Nottestaments nicht Erbin des Patienten geworden ist.

\section{Der Fall}

Die Testamentserrichtung

Ein Mitarbeiter des sozialmedizinischen Dienstes betreute 2006 einen schwerkranken Krebspatienten, der sich in stationärer Behandlung bei dessen Arbeitgeber, einem Universitätsklinikum (Beklagte) befand. Im Rahmen dieser Betreuung äußerte der Patient den Wunsch, ein Testament zugunsten seiner Lebensgefährtin, der Klägerin, errichten zu wollen. Zu einer Testamentserrichtung zu einem früheren Zeitpunkt war es nicht gekommen, da der Patient seine Krankheit bis zu seinem stationären Aufenthalt nicht ausreichend ernst genommen hatte und davon ausgegangen war, noch genügend Zeit zu haben, seine Angelegenheiten zu regeln. Nachdem ihm schließlich von ärztlicher Seite eindringlich sein Krankheitszustand klar gemacht worden war, wollte er seine Lebensgefährtin als Erbin einsetzen, um diese finanziell abzusichern. Aufgrund seiner bereits fortgeschrittenen Erkrankung war der Patient selbst jedoch nicht mehr in der Lage, ein Testament handschriftlich aufzusetzen, sodass der Krankenhausmitarbeiter einen Testamentsentwurf mit folgendem Wortlaut auf der Maschine tippte:

„Ich, (Name), z.Z. stationär in der Uniklinik X, im Vollbesitz meiner geistigen Kräfte, möchte, dass mein gesamtes Vermögen nach meinem Tod an meine alleinige Erbin und Lebenspartnerin, (Name) - geb. am...in...übergeht. Aufgrund meiner Krebserkrankung bin ich in meinen körperlichen Funktionen stark eingeschränkt. Auf meinen ausdrücklichen Wunsch wurde ich bei der Erstellung dieses Testaments seitens der Uniklinik X unterstützt. Um eventuelle Zweifel an meiner Geschäftsfähigkeit auszuräumen, beurkunden die untenstehenden Zeugen diese mit ihren Unterschriften.“

Dies wurde dem Patienten vom Verfasser in Gegenwart der Klägerin und einer Ärztin als Zeugin vorgelesen und anschlie- ßend vom Patienten selbst und als Zeugen vom Verfasser (Krankenhausmitarbeiter) und der Ärztin des Krankenhauses unterzeichnet. Dabei gingen die Beteiligten davon aus, ein formgültiges Nottestament errichtet zu haben. Erst danach willigte der Patient in eine Schmerztherapie mit Morphium ein und starb kurz darauf.

\section{Nottestament}

Im vorliegenden Fall ging es um die Frage, ob ein unwirksam errichtetes Nottestament eine Haftung des Krankenhausträgers auslösen kann, wenn Mitarbeiter von ihm an dessen Ausarbeitung beteiligt sind.

Grundsätzlich muss ein Testament, damit es Wirksamkeit erlangt, eigenhändig geschrieben und unterschrieben oder vom Notar errichtet sein. Die Errichtung eines Nottestaments ist nur dann zulässig, wenn der Testierende nicht mehr in der Lage ist, ein Testament selbst handschriftlich zu verfassen und auch kein Notar rechtzeitig zu erreichen ist. Diese Voraussetzungen können bei schwerkranken Patienten unter Umständen vorliegen.

\section{Ungültigkeit des Testaments}

Nach dem Tode ihres Lebensgefährten beantragte die Klägerin mit dem errichteten Nottestament einen Erbschein, dessen Erteilung jedoch abgelehnt wurde. Grund hierfür war, dass ein Nottestament gemäß § 2250 des Bürgerlichen Gesetzbuches (BGB) nur dann gültig ist, wenn unter anderem 3 unabhängige Zeugen bei der Verlesung des letzten Willens anwesend sind und dies anschließend mit ihrer Unterschrift dokumentieren. Die Lebensgefährtin selbst allerdings konnte keine Zeugin sein, da sie selbst in dem Testament begünstigt wurde. Somit wurde das Testament nur vor 2 unabhängigen Zeugen verlesen und unterschrieben. Die Klägerin wurde daher nicht Erbin des beträchtlichen Vermögens des Patienten, das unter anderem aus einem unbelaste- ten mit 2 Häusern bebauten Grundstück sowie einem Barvermögen in Höhe von mehr als 300000 Euro bestand. Daraufhin verlangte sie von dem Krankenhausträger das entgangene Erbe in Form von Schadensersatz (zunächst in einer Teilklage über einen Teilbetrag). In erster Instanz wurde die Klage vom Landgericht mit der Begründung abgewiesen, dass es sehr unwahrscheinlich erscheine, dass die Klägerin ohne die Pflichtverletzung des Krankenhausmitarbeiters Erbin geworden wäre. Das Oberlandesgericht sah dies jedoch anders und gab der Lebensgefährtin Recht.

Keine Pflicht zur Rechtsberatung aber Pflicht zur Unterstützung Der Krankenhausvertrag zwischen Patient und Krankenhausträger verpflichtet Letzteren zwar dazu, den Patienten in gewissen sozialen Bereichen $\mathrm{zu}$ betreuen und zu unterstützen, aber nicht dazu, dem Patienten Rechtsberatung zu erteilen. Somit traf den Mitarbeiter keine Verpflichtung, für den Patienten und dessen Lebensgefährtin ein Testament zu errichten. Allerdings ist der Krankenhausträger, und damit auch seine Mitarbeiter, verpflichtet, dem Patienten in gewissen sozialen Belangen Unterstützung zu gewähren. Von dieser Unterstützung umfasst sein kann nach Auffassung des Gerichts auch die Pflicht zur zumutbaren Unterstützung bei einer Testamentserrichtung, die aber nur soweit geht, als dass dem Patienten zu raten ist, einen Notar oder Rechtsanwalt hinzuzuziehen.

Verhinderung eines formwirksamen Testaments

Entscheidend in diesem Fall war, dass das Krankenhaus und seine Mitarbeiter im Rahmen ihrer Pflichten vor allem alles zu unterlassen haben, was die Errichtung eines formwirksamen Testaments behindern oder sogar verhindern könnte.

Daraus folgte, dass den Mitarbeiter ab dem Zeitpunkt, an dem er sich bereit erklärte, das Testament zu verfassen, auch die Pflicht traf, sich entsprechend sachkundig zu machen, um eine formunwirksame Erbeinsetzung zu vermeiden. Andernfalls ist seine Mitwirkung nämlich 
geeignet, die Errichtung eines formwirksamen Testaments zu gefährden oder zu verhindern.

Das Gericht ging, anders als die erste Instanz, davon aus, dass der Lebensgefährte der Klägerin zu einem späteren Zeitpunkt noch zu ihren Gunsten testiert hätte, insbesondere da die Schmerztherapie noch hätte hinausgezögert werden können und er somit auch noch länger in der Lage gewesen wäre, ein anderes Testament zu errichten. Jedoch vertrauten die Klägerin und ihr Lebensgefährte darauf, dass das von dem betroffenen Mitarbeiter verfasste Testament und von ihm vorgeschlagene Vorgehen geeignet war, die Klägerin wirksam als Erbin einzusetzen.

Berechtigtes Vertrauen des Patienten auf die Wirksamkeit des Testaments Das Verhalten des Mitarbeiters war auch geeignet, den Eindruck zu erwecken, dass er die erforderlichen Kenntnisse besäße, um ein formwirksames Nottestament zu errichten. So hat er einerseits, nach der Überzeugung der Richter, zu keinem Zeitpunkt darauf hingewiesen, dass ein Rechtsanwalt oder Notar hinzuzuziehen sei. Auch aus seiner Dokumentation der Gespräche mit dem Patienten und dessen Lebensgefährtin gehe dies nicht hervor, obwohl auch über die finanzielle Zukunft der Lebensgefährtin gesprochen wurde und auch der Verlauf der Aktivitäten bezüglich der Testamentserrichtung mit aufgenommen wurde.

Auch musste der Mitarbeiter einräumen, dass er selbst auf die Idee gekommen sei, dass Testament in der erfolgten Art und Weise zu erstellen, was nach Dafürhalten des Gerichts dafür spricht, dass er selbst von seiner Sachkunde überzeugt war. Auch habe er, nachdem klar war, dass das Testament unwirksam ist, nie darauf verwiesen, dass er zur Hinzuziehung eines Anwalts oder Notars geraten hätte, sondern sich ausschließlich dahingehend geäußert, weshalb das Testament nach seinem Dafürhalten wirksam sein müsste. Dies, sowie die Zeugenaussage der bei der Testamentsverlesung anwesenden Ärztin, dass kein Hinweis auf die fehlende Rechtskenntnis des sozialmedizinischen Mitarbeiters ergangen sei, sprechen dafür, dass ein Hinweis, dass die Einholung von Rechtsrat erforderlich sei, tatsächlich nie ergangen ist.

Des Weiteren habe er durch Äußerungen, wie es wäre „Gang und Gebe“, dass in so großen Krankenhäusern Nottestamente errichtet würden, die Überzeugung der
Klägerin und deren Lebensgefährten gestärkt, er hätte die entsprechenden Sachkenntnisse.

Auch dass der Patient erst nachdem das Testament abgefasst wurde in eine Schmerztherapie mit Morphium einwilligte, spricht dafür, dass er in dessen Gültigkeit vertraute und davon ausging, dass seine Angelegenheiten nunmehr geregelt seien.

Hätte der Mitarbeiter darauf hingewiesen, dass er aufgrund fehlender Rechtskenntnisse keine Gewähr für die Wirksamkeit übernehmen kann und zur Hinzuziehung eines Rechtsanwalts oder Notars geraten hätte, wäre das Urteil wohl anders ausgefallen, da dann unter Umständen nicht von einer Pflichtverletzung des Mitarbeiters ausgegangen worden wäre.

\section{Möglichkeit zur Errichtung eines}

anderen, formwirksamen Testaments hätte noch bestanden

Damit durch das Vorgehen des Mitarbeiters auch die Errichtung eines formwirksamen Testaments verhindert worden wäre, hätte es dem Patienten auch noch möglich gewesen sein müssen, ein tatsächlich gültiges Testament aufzusetzen. Zwar war er aufgrund seiner fortgeschrittenen Krankheit nicht mehr in der Lage, ein handschriftliches Testament aufzusetzen, allerdings gibt es nach Meinung des Gerichts keinen Grund, warum nicht ein formgültiges Nottestament entsprechend der dargestellten Voraussetzungen aufgesetzt hätte werden können oder ein notarielles Testament.

Nach Aussagen seines Arztes wäre es durchaus möglich gewesen, den Beginn der Schmerztherapie noch zu verschieben, sodass davon auszugehen war, dass der Patient dies auch getan hätte, um weitere Vorkehrungen zugunsten seiner Lebensgefährtin zu treffen, wäre er der Überzeugung gewesen, kein wirksames Testament hinterlassen zu haben.

Auch folgte das Gericht nicht dem Vorbringen der Beklagten, dass eine Errichtung eines notariellen Testaments schon aufgrund der Abneigung des Patienten gegen Rechtsanwälte und Notare unterblieben wäre.

Krankenhausaufnahmevertrag als Grundlage für den Schadensersatzanspruch

Als Grundlage für die Haftung des Krankenhausträgers hat das Gericht den Krankenhausvertrag zwischen dem Lebensge- fährten der Klägerin und der Beklagten herangezogen.

In die Schutzpflicht, die aufgrund des Krankenhausvertrages zunächst gegenüber dem Patienten bestand, war auch seine Lebensgefährtin mit einbezogen, obwohl sie selbst nicht Partei des Vertrages war.

Grundlage hierfür ist die juristische Konstellation des sogenannten Vertrages mit Schutzwirkung zugunsten Dritter, im Rahmen dessen ein eigentlich nicht am Vertrag Beteiligter, wie hier die Lebensgefährtin des Patienten, in den Schutzbereich eines Vertrages mit einbezogen wird. Voraussetzung hierfür ist, dass der Dritte bestimmungsgemäß mit der Leistung des Schuldners, hier dem Krankenhaus, in Berührung kommt, der Gläubiger, hier der Lebensgefährte, ein besonderes Interesse an der Einbeziehung des Dritten hat und dies auch für den Schuldner erkennbar war. Schließlich muss an der Erstreckung der Schutzpflichten ein Bedürfnis bestehen, weil der Dritte (hier die Klägerin) andernfalls nicht ausreichend geschützt wäre. Als gewünschte Erbin des Patienten kam die Lebensgefährtin mit der Pflicht des Krankenhausträgers und seiner Mitarbeiter, die Errichtung eines formwirksamen Testaments nicht zu erschweren oder zu behindern, bestimmungsgemäß genauso in Berührung wie der Patient selbst, auch trifft sie eine Verletzung dieser Pflicht gleichermaßen. Der Patient wiederum hatte auch ein Interesse daran, dass seine Lebensgefährtin in den Schutzbereich mit einbezogen würde.

Dies war für den Krankenhausmitarbeiter nach Auffassung des Gerichts auch erkennbar, da die Klägerin im Rahmen der Behandlung ihres Lebensgefährten in einem Vertrauensverhältnis zu den Mitarbeitern des Krankenhauses stand, insbesondere auch zu dem betroffenen Mitarbeiter. Sie war auch mit in Diskussionen über die Angelegenheiten ihres Lebensgefährten einbezogen. Da auch ein anderweitiger Anspruch der Klägerin aus dem hier zugrunde liegenden Sachverhalt nicht ersichtlich war, war sie auch schutzbedürftig.

Der Krankenhausträger muss sich das Verhalten seines Mitarbeiters (Errichtung eines formunwirksamen Testaments) als sogenannter Erfüllungsgehilfe zurechnen lassen. Der Krankenhausträger wurde schließlich zu einer Teilzahlung von zunächst 78931,37 Euro nebst Zinsen verurteilt. 


\section{Fazit}

Die Entscheidung ist in zweierlei Hinsicht von Bedeutung. Zum einen macht sie sehr deutlich, dass die Einbeziehung von Angehörigen und nahe stehenden Personen der Patienten in den Pflichtenkreis des Krankenhauses und seiner Mitarbeiter im Rahmen des Krankenhausvertrages dazu führen kann, dass diese auch die Verletzung von Nebenpflichten geltend machen können.

Zum anderen darf angesichts der Höhe der möglichen Schadensersatzforderungen sowie der Vielzahl von Fehlern, die bei der Errichtung eines Nottestaments unterlaufen können, das erhebliche Haftungsrisiko für den Krankenhausträger nicht unterschätzt werden.

Ausgehend von dem vorliegenden Urteil ist ein Krankenhaus daher gut beraten, wenn es seine Mitarbeiter anweist, bei Anfragen von Patienten nach einem Testament auf die Einholung von Rechtsrat durch einen Rechtsanwalt oder Notar zu verweisen und nicht selbst an der Errichtung des Testaments mitzuwirken. Dieser erteilte Hinweis sollte in jedem Fall in die Dokumentation (am besten mit Datum, Uhrzeit, Zeugen) aufgenommen werden. Die Errichtung eines (Not-)Testaments sollte jedenfalls nicht selbst in die Hand genommen werden. 